\title{
Thousand Organisms per Gram
}

National Cancer Institute

\section{Source}

National Cancer Institute. Thousand Organisms per Gram. NCI Thesaurus. Code C71190.

A unit of measure of organism content expressed in thousands of organisms per unit of mass equal to one gram. 